\title{
Initiative pour la transparence
}

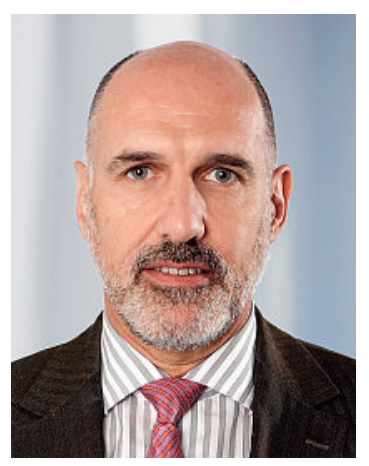

Si vous ne le saviez pas encore, vous le lirez à la page 437 de ce Bulletin: les médecins des trois régions linguistiques de la Suisse, soutenus par la FMH, ont lancé l'automne dernier une initiative populaire fédérale qui vise à imposer une séparation complète entre l'assurance-maladie sociale obligatoire d'une part, et les assurances complémentaires privées d'autre part. Ainsi, les caisses-maladie ne pourront plus proposer ces deux types d'assurance, qui relèvent de règles légales différentes et induisent des conflits d'intérêt majeurs. Elles devront opter pour l'une ou pour l'autre de ces activités.

A juste titre, la loi prévoit que les caisses-maladie doivent être des entreprises sans but lucratif, car ce sont des institutions mandatées par l'Etat pour mettre en œuvre l'assurance-maladie sociale de base. L'idée est évidemment d'éviter ainsi les distorsions qui apparaissent inévitablement dans le domaine de la santé lorsque les lois du marché et la concurrence économique y interviennent de façon incontrôlée, ou excessive.

\section{La conjonction de l'assurance sociale et \\ d'assurances privées sous un même chapeau ne devrait simplement plus être possible.}

Or la loi actuelle laisse ouverte la possibilité de mélanger les genres et la tentation est parfois trop forte! La conjonction de l'assurance sociale et d'assurances privées sous un même chapeau ne devrait simplement plus être possible, car soit par la présence «à l'arrière-plan» de groupes d'assureurs privés, soit parce que les caisses-maladie ont elles-mêmes souhaité élargir leur offre à l'assurance complémentaire, on se trouve dans une situation qui souvent pervertit le système.

C'est ainsi que, sélection des risques oblige et selon que vous serez jeune ou vieux, bien-portant ou malade, vous pourrez changer de caisse-maladie en trois clics de souris, ou alors votre téléphone sera «mis en attente», votre lettre se perdra, et la réponse n'arrivera jamais. De même pour la rapidité des remboursements, le tiers-payant en pharmacie, etc.; l'existence de fuites d'informations confidentielles entre ordinateurs sera laissée à l'appréciation de chacun-e, selon ses expériences ...

Cette chasse aux bons risques, ces manœuvres de tri des assurés, ainsi des flux financiers que l'on peine parfois à comprendre (comme au sujet des activités politiques de santésuisse), sont inacceptables; ils pervertissent notre système et doivent être interdits. C'est le but de l'initiative populaire «pour la transparence de l'assurance-maladie» dont il est question dans cette édition du BMS, et c'est pour cela que l'Assemblée des délégués de la FMH a décidé de la soutenir et de vous inviter à le faire aussi.

\section{Vous êtes appelés, toutes et tous, à signer et à faire signer l'initiative des médecins «pour la transparence de I'assurance-maladie».}

Reste cependant une question à aborder, c'est celle de la simultanéité de plusieurs initiatives.

En effet, l'initiative «pour une caisse-maladie publique et unique» vient aussi d'être lancée par la gauche, par les associations de patients, et par une quantité d'associations professionnelles du milieu de la santé. La FMH n'a pas encore pris position sur cette initiative. Les auteurs romands de l'initiative sur la transparence, celle dont il est question ici, ne pensent pas que les initiatives se concurrencent, et ils poussent à les soutenir les deux.

Et il faut évoquer aussi le projet des cantons de Suisse orientale visant à des caisses cantonales ou régionales; ce projet n'est pas encore formulé mais déjà bien présent dans le débat.

Qu'en penser? On doit savoir en réalité que la discussion sur ces initiatives, au Parlement, se fera certainement dans le sens d'une convergence entre plusieurs options, plusieurs variantes, qui auront leurs racines dans les textes en question. Il ne devrait donc pas y avoir concurrence mais bien complémentarité entre ces diverses possibilités d'évolution de notre système d'assurance-maladie.

C'est ainsi sans la moindre hésitation que vous êtes appelés, toutes et tous, à signer et à faire signer l'initiative des médecins «pour la transparence de l'assurance-maladie»à la signer vous-même et à la faire signer largement autour de vous. Avec le Comité d'initiative*, nous vous y encourageons vivement et vous en remercions.

Dr Jacques de Haller, Président de la FMH

\footnotetext{
* Bertrand Buchs, Franco Denti, Charles Abram Favrod-Coune, Marc-Henri Gauchat, Marcus Lütolf, Jürg Nadig, Remo Osterwalder, Jean-Pierre Pavillon, Pierre-Alain Schneider, Urs Stoffel, Igor Svarin, Pierre Vallon, Paul Olivier Vallotton, Hans-Anton Vogel, Peter Wiedersheim
} 